\title{
Core-shell silk hydrogels with spatially tuned conformations as drug-delivery system
}

\author{
Le-Ping Yan $^{1,2}$, Joaquim M. Oliveira ${ }^{1,2 *}$, Ana L. Oliveira ${ }^{1,2,3}$ and Rui L. Reis ${ }^{1,2}$ \\ ${ }^{1} 3$ B's Research Group - Biomaterials, Biodegradables and Biomimetics, University of Minho, Headquarters of the European Institute of \\ Excellence on Tissue Engineering and Regenerative Medicine, Guimarães, Portugal \\ ${ }^{2}$ ICVS/3B's - PT Government Associate Laboratory, Braga/Guimarães, Portugal \\ ${ }^{3} \mathrm{CBQF}$ - Center for Biotechnology and Fine Chemistry, School of Biotechnology, Portuguese Catholic University, Porto, Portugal
}

\begin{abstract}
Hydrogels of spatially controlled physicochemical properties are appealing platforms for tissue engineering and drug delivery. In this study, core-shell silk fibroin (SF) hydrogels of spatially controlled conformation were developed. The core-shell structure in the hydrogels was formed by means of soaking the preformed (enzymatically crosslinked) random coil SF hydrogels in methanol. When increasing the methanol treatment time from 1 to $10 \mathrm{~min}$, the thickness of the shell layer can be tuned from about 200 to about $850 \mu \mathrm{m}$ as measured in wet status. After lyophilization of the rehydrated core-shell hydrogels, the shell layer displayed compact morphology and the core layer presented porous structure, when observed by scanning electron microscopy. The conformation of the hydrogels was evaluated by Fourier transform infrared spectroscopy in wet status. The results revealed that the shell layer possessed dominant $\beta$-sheet conformation and the core layer maintained mainly random coil conformation. Enzymatic degradation data showed that the shell layers presented superior stability to the core layer. The mechanical analysis displayed that the compressive modulus of the core-shell hydrogels ranged from about $25 \mathrm{kPa}$ to about $1.1 \mathrm{MPa}$ by increasing the immersion time in methanol. When incorporated with albumin, the core-shell SF hydrogels demonstrated slower and more controllable release profiles compared with the non-treated hydrogel. These core-shell SF hydrogels of highly tuned properties are useful systems as drug-delivery system and may be applied as cartilage substitute. Copyright (C) 2016 John Wiley \& Sons, Ltd.
\end{abstract}

Received 25 January 2016; Revised 16 April 2016; Accepted 19 April 2016

Keywords silk fibroin; core-shell hydrogel; spatially controlled conformation; drug delivery; beta-sheet; random coil; tissue engineering and regenerative medicine

\section{Introduction}

Due to the possibility of tuning their physicochemical properties to mimic the extracellular matrix (ECM), hydrogels have been applied in medical devices fabrication, tissue engineering and regenerative medicine (TERM), and drug-delivery systems (Seliktar, 2012). Typically, hydrogels present homogeneous characteristics and lack of sufficient spatial control in their properties (Kloxin et al., 2009). Therefore, the development of hydrogels with spatiotemporally controlled properties constitutes a great demand in drug-delivery systems. Studies have been performed to address this great challenge by development of multi-layer hydrogels coating (Choi et al., 2012), core-shell hydrogel microspheres consisting of temperature-sensitive cores and pH-sensitive shells (Ma et al., 2010), and core-shell self-assembling peptides hydrogel (Koutsopoulos and Zhang, 2012).

Besides those interesting approaches that have been proposed, the biological systems also provide useful hints

*Correspondence to: Joaquim M. Oliveira, 3B's Research Group - Biomaterials, Biodegradables and Biomimetics, University of Minho, Headquarters of the European Institute of Excellence on Tissue Engineering and Regenerative Medicine, AvePark, Taipas, Guimarães, 4806-909, Portugal. E-mail: miguel.oliveira@dep. uminho.pt on spatially tuning the properties of hydrogels via varying the spatial arrangements of biomacromolecules (Fratzl et al., 1998). The heterogeneous properties of the tissues are a consequence of the distinct orientations of the biomacromolecules constituting its ECM. For example, cartilage is mainly composed of collagen type II, in its four zones (Pearle et al., 2005). The compressive modulus of the deeper zone is much higher than the superficial zone, partially due to the different orientations of collagen fibrils in these two areas. Additionally, it is possible to envision controlling the bulk properties of the hydrogels by means of tuning the molecule conformation (Nowak et al., 2002). Therefore, the spatial modulation of the hydrogel properties can also be envisioned by controlling the spatial conformation or orientation of the macromolecules in the hydrogel network. For instance, multimembrane chitosan hydrogels were developed by repeating the interrupted-neutralization of chitosan alcohol gels in sodium hydroxide solution (Ladet et al., 2008). The inter-membrane space in the hydrogels was induced by the distinct charge status of the alcohol gel and the neutralized gel.

Inspired by the biological systems and abovementioned studies, we hypothesized that protein-based hydrogels of spatial tuned properties can be prepared by modulating their conformations in space and applied as novel drug-delivery systems. Among the common protein 
biomaterials, silk fibroin (SF) derived from Bombyx mori cocoon has consolidated its application in the TERM field due to its outstanding compatibility with cells and tissues (Kundu et al., 2014; Silva et al., 2014; Vepari and Kaplan, 2007). Mainly, three kinds of conformations have been identified in SF (Jin and Kaplan, 2003). SF in the spun silk fibre contains dominant insoluble $\beta$-sheet (Silk-II, antiparallel $\beta$-pleated) conformation, which confers the high strength and toughness to the fibres. Random coil exists in regenerated aqueous SF solution of low concentration. Silk-I ( $\alpha$-form, type II $\beta$-turn) can be found in highconcentration SF solution without physical shear, such as silk dope in the gland before spinning.

Silk fibroin hydrogels have showed great promise in controlled drug delivery (Numata et al., 2012). For example, anti-cancer drug doxorubicin had been incorporated into SF hydrogels for the treatment of primary breast cancer (Seib et al., 2013). SF hydrogels can be obtained by means of decreasing $\mathrm{pH}$ value or increasing the temperature of SF solution, or the addition of chemical compounds or applying external physical stimulus to the SF solution (Kim et al., 2004; Motta et al., 2004; Wang et al., 2008; Yucel et al., 2009). SF hydrogels reported by the aforementioned methods presented dominant $\beta$-sheet conformation. Electrical stimulus was also able to generate SF adhesive gels with dominant random coil conformation (Leisk et al., 2010). Still, these gels presented a lack of mechanical stability (Lin et al., 2013). Recently, our group developed injectable and stimuli-responsive SF hydrogels with dominant random coil conformation via peroxidase-mediated crosslinking (Yan et al., 2016). Partlow et al. also reported an important work on SF hydrogels using the same strategy as the one that has been previously patented by our group (Partlow et al., 2014; Reis et al., 2014).

Here, we report a facile method to prepare SF hydrogels with spatially controlled conformation, and study their potentials as drug-delivery systems. To the best of our knowledge, SF hydrogels with spatially controlled conformation have not been reported elsewhere.

\section{Materials and methods}

\subsection{Materials}

Cocoons of Bombyx mori were purchased from the Portuguese Association of Parents and Friends of Mentally Disabled Citizens (APPACDM, Castelo Branco, Portugal). If not mentioned otherwise, all the materials or reagents were purchased from Sigma-Aldrich (St Louis, MO, USA).

\subsection{Preparation of the core-shell SF hydrogels}

Silk fibroin was purified by degumming of silk cocoon in $0.02 \mathrm{~mol} / \mathrm{l}$ sodium carbonate boiling solution for $1 \mathrm{~h}$ (Yan et al., 2012, 2015). SF was first dissolved in 9.3 mol/1 lithium bromide solution, and then transferred into a benzoylated dialysis tubing (molecular weight cut-off: 2 $\mathrm{kDa}$ ) and dialysed in distilled water for $48 \mathrm{~h}$. Afterwards, the SF solution was dialysed against phosphate-buffered saline solution (PBS, without calcium and magnesium ions) for $12 \mathrm{~h}$, and subsequently concentrated in $20 \mathrm{wt} \%$ poly(ethylene glycol) solution. The final concentration of the SF aqueous solution was tested by drying the SF solution in an oven at $70{ }^{\circ} \mathrm{C}$ overnight. The SF solution was diluted into $16 \mathrm{wt} \%$ by the addition of PBS. The random coil SF hydrogels were obtained via horseradish peroxidase (HRP; type VI, $260 \mathrm{U} / \mathrm{mg}$ solid) mediated gelation (Reis et al., 2014). Briefly, $1 \mathrm{ml}$ SF solution was mixed with $50 \mu \mathrm{l} \mathrm{HRP} \mathrm{solution}(0.84 \mathrm{mg} / \mathrm{ml})$ and $65 \mu \mathrm{l}$ hydrogen peroxide solution $(0.36 \mathrm{wt} \%)$, followed by transferring $200 \mu \mathrm{l}$ of the mixture into a polypropylene mould (diameter: $8 \mathrm{~mm}$ ) and placing the moulds into the $37^{\circ} \mathrm{C}$ oven until the gel formed. The SF hydrogel discs were removed from the moulds and used for the preparation of the core-shell SF hydrogels. The core-shell SF hydrogels were prepared by immersion of the prepared random coil SF hydrogel discs in methanol for 1, 3, 5 and $10 \mathrm{~min}$. At the end of each time point, the hydrogel discs were removed from the methanol and washed in PBS three times to eliminate the organic solvent. After the methanol treatment, the hydrogel disc formed a core-shell structure compromising a stiff opaque shell layer, a soft transparent core domain, and a very thin soft opaque interface region between the shell layer and the core domain.

\subsection{Measurement of the thickness}

The prepared core-shell SF hydrogel discs were longitudinally cut, and the soft inner region was separated from the stiff shell layer. The thickness of the wall in the shell layers of core-shell SF hydrogels was measured by means of using a micrometre. Three areas in one disc were measured and the values were averaged. For each group of discs, at least four specimens were tested.

\subsection{Scanning electron microscopy (SEM) observation}

The specimens were also analysed under a SEM (Nova NanoSEM 200; FEI, Hillsboro, OR, USA). The core-shell and non-treated hydrogels were longitudinally cut, followed by frozen in liquid nitrogen for $20 \mathrm{~min}$ and then at $-80{ }^{\circ} \mathrm{C}$ for at least $3 \mathrm{~h}$. Afterwards, the samples were lyophilized and observed by SEM. Before SEM evaluation, the samples were coated with Au/Pd SC502-314B in an evaporator coater (E6700; Quorum Technologies, East Grinstead, UK).

\subsection{Fourier-transform infrared spectroscopy (FTIR) analysis}

The conformation of the different domains in the core-shell SF hydrogels was characterized by FTIR in attenuated total reflectance (ATR) model (IRPrestige-21; 
Shimadzu, Kyoto, Japan). The samples after methanol treatment were washed and immediately tested by ATR-FTIR. The tested domains were the external surface of the shell layer, the inner surface of the shell layer, the interface area between the shell and the core region, and the core region. The samples were analysed by contacting the germanium crystal in the FTIR. Each specimen was scanned 48 times from 500 to $4000 \mathrm{~cm}^{-1}$ with a resolution of $4 \mathrm{~cm}^{-1}$, in wet state. Silk solution and hydrogels without methanol treatment were used as controls. PBS was scanned as background. Three specimens were analysed in each group.

\subsection{Enzymatic degradation}

The shell layer of the core-shell SF hydrogel was degraded in protease XIV solution. The as-prepared SF hydrogel and the core region of the core-shell SF hydrogels, which were immersed in methanol for $10 \mathrm{~min}$, were also tested. About $50 \mathrm{mg}$ hydrogel (wet weight after removing surface liquid by filter paper) was immersed in $5 \mathrm{ml}$ protease XIV solution and kept in a thermostatic water bath at $37^{\circ} \mathrm{C}$. The enzyme solutions of $0.2 \mathrm{U} / \mathrm{ml}$ and $0.005 \mathrm{U} / \mathrm{ml}$ were used for the shell layer and the core region, respectively. The samples were degraded for 1, 2, 4, 6 and $12 \mathrm{~h}$, and the weight loss ratio was calculated as follows:

$$
\text { Weight loss ratio }(\%)=\left(W_{\mathrm{i}}-W_{\mathrm{t}}\right) / W_{\mathrm{i}} * 100 \%
$$

where $W_{\mathrm{i}}$ means the initial wet weight of the hydrogel and $W_{\mathrm{t}}$ is the wet weight tested at each time point. Four specimens were used for each group hydrogel.

\subsection{Hydration degree}

The hydration degree of the shell layer and the core domain of the hydrogels were evaluated. The shell layer and the core layer of the hydrogels were, respectively, immersed in PBS for $1 \mathrm{~h}$, and then their wet weights were recorded after removing the surface liquid by filter paper. Afterwards, the samples were dried at $70^{\circ} \mathrm{C}$ in an oven overnight. The dry weight of each sample was measured. The hydration degree was defined as follows:

$$
\text { Hydration degree }(\%)=\left(W_{\mathrm{i}}-W_{\mathrm{d}}\right) / W_{\mathrm{d}} * 100 \%
$$

where $W_{\mathrm{i}}$ refers to the initial wet weight of the sample and $W_{\mathrm{d}}$ is the dry weight of the sample. Four specimens were screened in each group.

\subsection{Compressive test}

The compressive modulus of the core-shell SF hydrogels was determined using a universal testing machine (Instron 4505, Instron, Norwood, MA, USA). The specimens were tested in an unconfined compression model, after removing the surface liquid by filter paper. The compressive rate was set at $2 \mathrm{~mm} / \mathrm{min}$ until reaching $50 \%$ strain. The modulus was determined from the slope of the initial linear domain in the compressive curve. At least six specimens were examined for each group.

\subsection{Albumin release profile in the core-shell SF hydrogels}

The albumin-fluorescein isothiocyanate conjugate (albumin-FITC) was used as a model drug to study the release profile from the core-shell SF hydrogels. The random coil hydrogel discs were first hydrated in PBS for $1 \mathrm{~h}$ after preparation, followed by immersion in $100 \mu \mathrm{g} / \mathrm{ml}$ albumin-FITC solution overnight at room temperature ( $1.5 \mathrm{ml} /$ disc). Afterwards, the hydrogel discs were removed from the albumin-FITC solution and rinsed in PBS. The SF hydrogels discs were used to prepare the core-shell SF hydrogels by immersion in methanol for 1 , 3,5 and $10 \mathrm{~min}$. The albumin-FITC release profiles of the as-prepared SF hydrogel and core-shell SF hydrogels were evaluated by immersion of each specimen in $4 \mathrm{ml}$ PBS. Due to the low amount of albumin incorporation in the core-shell SF hydrogel specimens with methanol treatment for 5 and $10 \mathrm{~min}, 2 \mathrm{ml}$ PBS was used for each disc in these two groups. The release of albumin-FITC was tested at $2,4,6,24,48,72,120$ and $168 \mathrm{~h}$. At each time point, the supernatant from each specimen was removed and an equal volume of fresh PBS was added. For the quantification of the released albumin-FITC, the fluorescence intensity of $100 \mu$ l supernatant of the removed PBS was read by a microplate reader (Synergy HT, Bio-Tek, VT, USA), with the excitation wavelength at $485 / 20 \mathrm{~nm}$ and the emission wavelength at $528 / 20 \mathrm{~nm}$. The samples without albumin-FITC incorporation were used as controls. The amount of incorporated albumin-FITC was used for the calculation of incorporation efficiency of the core-shell hydrogels groups. Five specimens were used for each group. For the determination of the total albumin-FITC in the hydrogels, the discs were immersed in $4 \mathrm{ml} \mathrm{PBS}$ and the supernatants were analysed periodically.

\subsection{Statistical analysis}

The data were presented by mean \pm standard deviation (SD). The results were analysed by one-way analysis of variance (ANOVA). The mean values between two groups were compared by Tukey's test. The significance level was ${ }^{* * * *} p<0.0001$.

\section{Results}

\subsection{Preparation of the core-shell hydrogels}

In this study, SF hydrogels of core-shell structure were prepared by immersion of the random coil SF hydrogels, 
which were preformed via peroxidase-mediated crosslinking, into methanol. The choice of methanol for the current study was because it can effectively induce fast transition from random coil to $\beta$-sheet in SF solution or film (Jin et al., 2005; Nazarov et al., 2004). Figure 1a showed the macroscopic appearance of the core-shell SF hydrogels. It was found that the outward appearance of the non-treated hydrogel was transparent, while hydrogel after methanol treatment became opaque (Figure 1a, top). The longitudinal section of the hydrogels after methanol treatment clearly displayed a core-shell structure (Figure 1a, top). The core layer and the shell layer integrated well, without an obvious interface (Figure 1a, bottom). The shell layer was opaque, while the core domain was transparent even after methanol treatment for $10 \mathrm{~min}$. The thickness of the wall in the shell layer increased from about $200 \mu \mathrm{m}$ to about $850 \mu \mathrm{m}$ (wet status) as enhancing the immersion time in methanol from 1 to $10 \mathrm{~min}$ (Figure $1 \mathrm{~b}$ ).

The morphology of the core-shell SF hydrogels was studied by SEM after rehydration and lyophilization of the methanol-treated hydrogels. A compact structure was observed in the shell layer of the methanol-treated SF hydrogels (Figure 1c). It was revealed that the wall thickness of the shell layer increased from about $100 \mu \mathrm{m}$ to about $500 \mu \mathrm{m}$ when increasing the methanol treatment time from 1 to $10 \mathrm{~min}$ (Figure 1c). No core-shell structure was observed in the non-treated SF hydrogel. The core region of the hydrogels demonstrated loose and porous structures, with a tendency to form sheet-like structure with increasing methanol treatment time. The external surface of the core-shell hydrogels was smooth, while that of the non-treated hydrogels was porous and rough.

\subsection{Spatially controlled conformation}

The conformation difference between the core region and the shell layer was studied by ATR-FTIR (Figure 2). It was found that the spectra of the aqueous SF solution, the non-treated SF hydrogel and the core region of the treated hydrogels all presented main absorption at about $1648 \mathrm{~cm}^{-1}$ (Figure 2a). A small shoulder located at $1627 \mathrm{~cm}^{-1}$ appeared when immersing the hydrogel in methanol for $10 \mathrm{~min}$; however it was not dominant. It has been reported that the peak located between 1640 $\mathrm{cm}^{-1}$ and $1650 \mathrm{~cm}^{-1}$ indicates the random coil conformation of SF, while the peak located at $1627 \mathrm{~cm}^{-1}$ is assigned to $\beta$-sheet conformation (Asakura et al., 1985; Leisk et al., 2010; Makaya et al., 2009). Figure 2a clearly showed that the dominant conformation in the aqueous silk solution, the non-treated SF hydrogel and the core region of the treated hydrogels was random coil. After

\section{a}
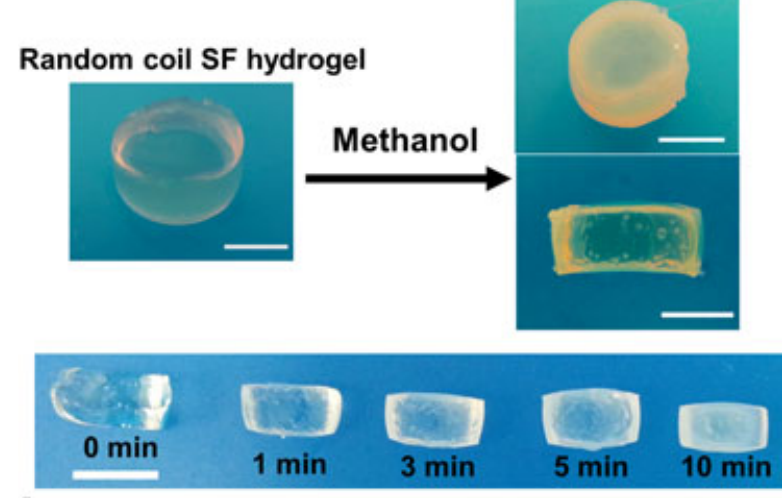

b

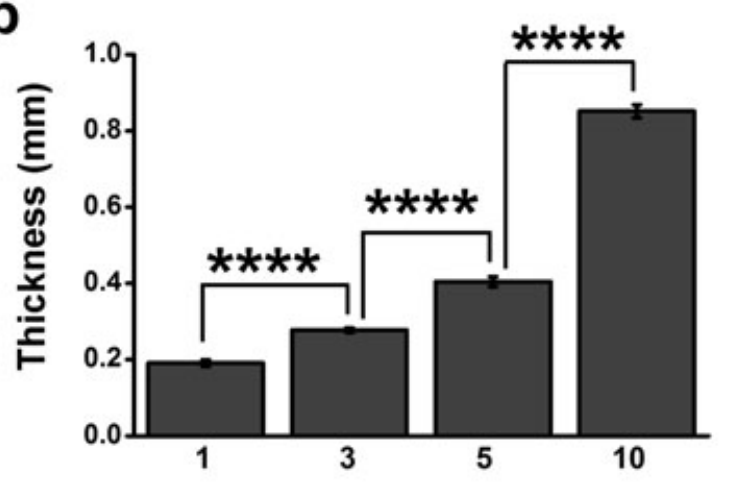

C

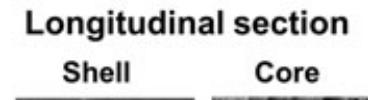

$0 \mathrm{~min}$
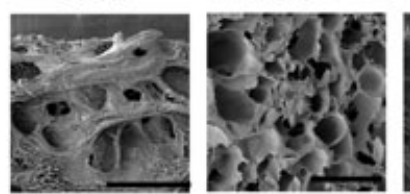

Outer surface

$1 \mathrm{~min}$
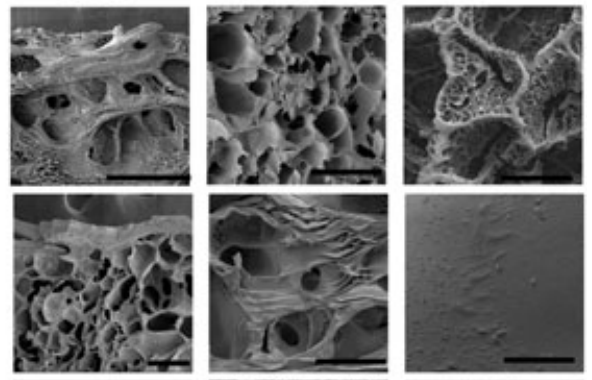

$3 \mathrm{~min}$
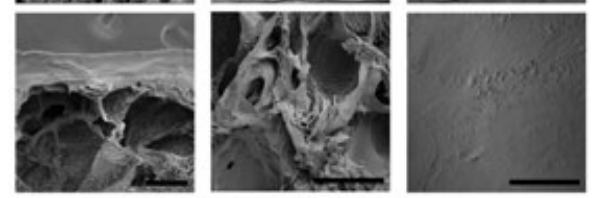

$5 \mathrm{~min}$
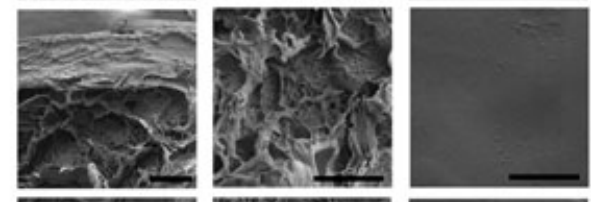

$10 \min$
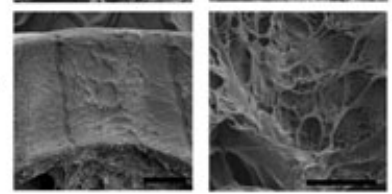

Immersion time (minute)

Figure 1. Silk fibroin (SF) hydrogels with core-shell structure. (a) Core-shell hydrogel formation by immersion of the random coil SF hydrogel in methanol. Top left: random coil SF hydrogel; top right: the outward appearance and longitudinal section of core-shell SF hydrogel after immersion in methanol for $3 \mathrm{~min}$. Bottom (from left to right): longitudinal section of the SF hydrogels after immersion in methanol for 0-10 min, showing core-shell structure. Scale bar: $5 \mathrm{~mm}$. (b) Thickness of the wall in the shell layer of the core-shell SF hydrogels after immersion in methanol for 1 up to10 min. Indicated statistically significant $(p<0.05)$. (c) The scanning electron micrograph (SEM) images of the core-shell SF hydrogels. Scale bar: 200: $\mu \mathrm{m}$ 

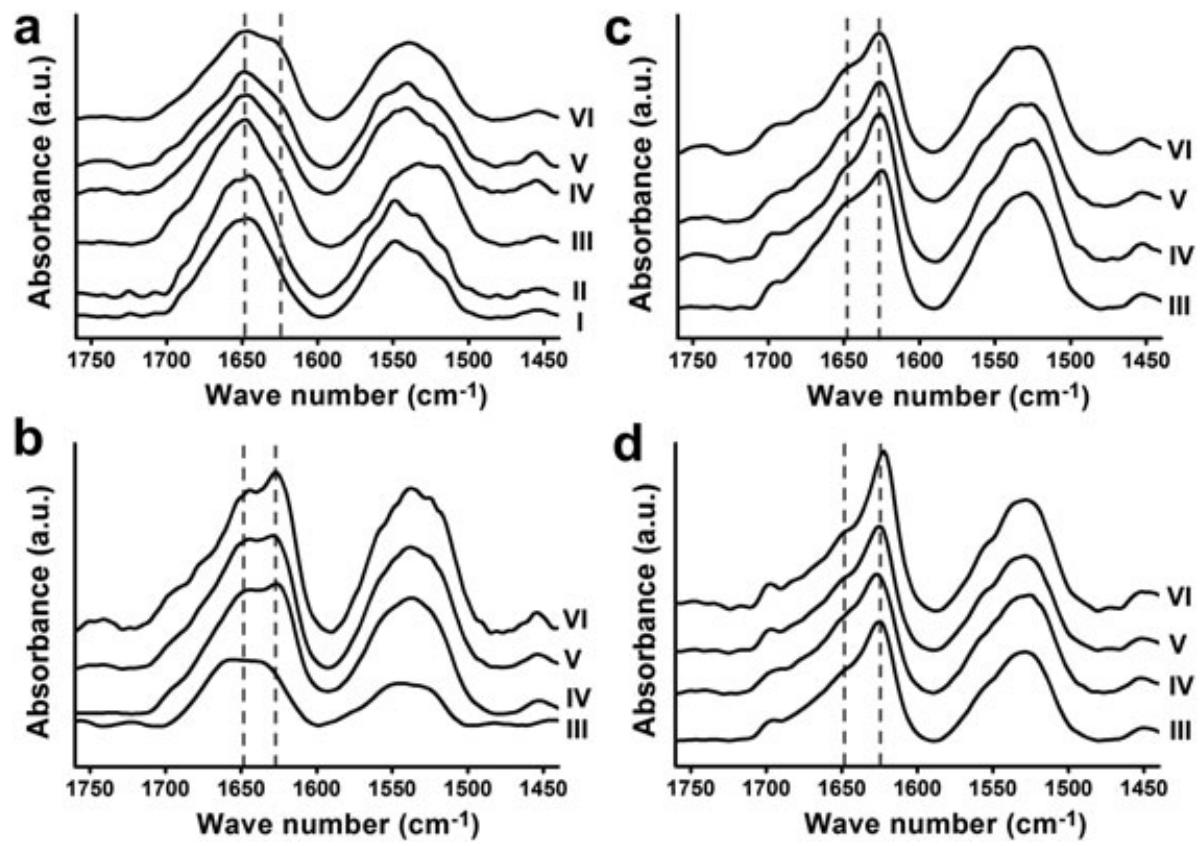

Figure 2. Attenuated total reflectance (ATR)-fluorescein isothiocyanate (FTIR) spectra of the core-shell silk fibroin (SF) hydrogels. (a) Core region, (b) the interface region, (c) the inner side of the shell layer, and (d) the external side of the shell layer of the core-shell SF hydrogels. (a) I and II are corresponding to SF solution and SF hydrogels without methanol treatment, respectively. (a-d) III, IV, V and VI are corresponding to the core-shell hydrogels after immersion in methanol for $1,3,5$ and 10 min, respectively

immersion in methanol for $10 \mathrm{~min}$, the core region presented a small amount of $\beta$-sheet conformation.

In the interface region, two strong peaks located at $1648 \mathrm{~cm}^{-1}$ and $1627 \mathrm{~cm}^{-1}$ appeared in all the spectra (Figure 2b), indicating that both the random coil and $\beta$-sheet conformations were dominant in this transitional region. The intensity of the peak located at $1627 \mathrm{~cm}^{-1}$ gradually increased by means of increasing the soaking time in methanol. When immersion in methanol was for more than $3 \mathrm{~min}$, the peak located at $1627 \mathrm{~cm}^{-1}$ showed slightly higher intensity than the one located at $1648 \mathrm{~cm}^{-1}$.

The spectra of the inner side of the shell layers all showed a strong peak located at $1627 \mathrm{~cm}^{-1}$ and an obvious shoulder peak located at $1648 \mathrm{~cm}^{-1}$ (Figure 2c). The spectra of the external surface of the shell layers exhibited a main peak located at about $1627 \mathrm{~cm}^{-1}$ and a tiny shoulder peak located at $1648 \mathrm{~cm}^{-1}$ (Figure 2d). The absorptions at $1627 \mathrm{~cm}^{-1}$ in the spectra of the external surface of the shell layer were sharper than the ones observed in the spectra of the inner side of the shell layer. These results demonstrated that the external side of the shell layer possessed a superior amount of $\beta$-sheet conformation and less amount of random coil contents compared with the inner side of the shell layer. The ATRFTIR analysis clearly showed the spatial conformation transition and distribution in the core-shell SF hydrogels.

\subsection{Degradation profile and hydration degree}

Figure 3a showed the enzymatic degradation profiles of the non-treated hydrogels and the core layer of the coreshell hydrogels. In each tested time point, these two groups of hydrogels presented similar weight loss profiles and both degraded completely within $12 \mathrm{~h}$. The degradation of the shell layer of the core-shell hydrogels was performed in protease solution of higher concentration (39 times higher) than the one used for non-treated hydrogels. The shell layers that had been treated by methanol for 3, 5 and 10 min showed 20\%, 10\% and 5\% weight loss within $12 \mathrm{~h}$, respectively (Figure $3 \mathrm{~b}$ ). However, the shell layer immersed in methanol for 1 min degraded completely within $4 \mathrm{~h}$. After degradation for more than $4 \mathrm{~h}$, there were only slight increases of the weight loss ratios in the shell layer of the groups treated by methanol for 3 and $5 \mathrm{~min}$. No obvious mass loss after $2 \mathrm{~h}$ was recorded for the shell layer that had been immersed in methanol for $10 \mathrm{~min}$. The weight remaining scores of the shell layer in the core-shell hydrogels were summarized in Table 1. Regarding the hydration test, the core region of the core-shell hydrogels presented a similar hydration degree compared with the non-treated hydrogels (Figure 3c). There were no significant differences in the hydration degree of the shell layers of the methanol-treated groups. However, the hydration degrees of the shell layer were much less than that of the core region (Figure 3c).

\subsection{Mechanical properties}

The mechanical properties of the core-shell SF hydrogels improved dramatically when increasing the methanol treatment time (Figure 3d). The non-treated random coil SF hydrogels showed a compressive modulus at about 22 $\mathrm{kPa}$. After immersion in methanol for $10 \mathrm{~min}$, the compressive modulus of the core-shell SF hydrogels increased more than 50 times $(\sim 1.12 \mathrm{MPa})$ compared with that of the non-treated group. The improvement of 

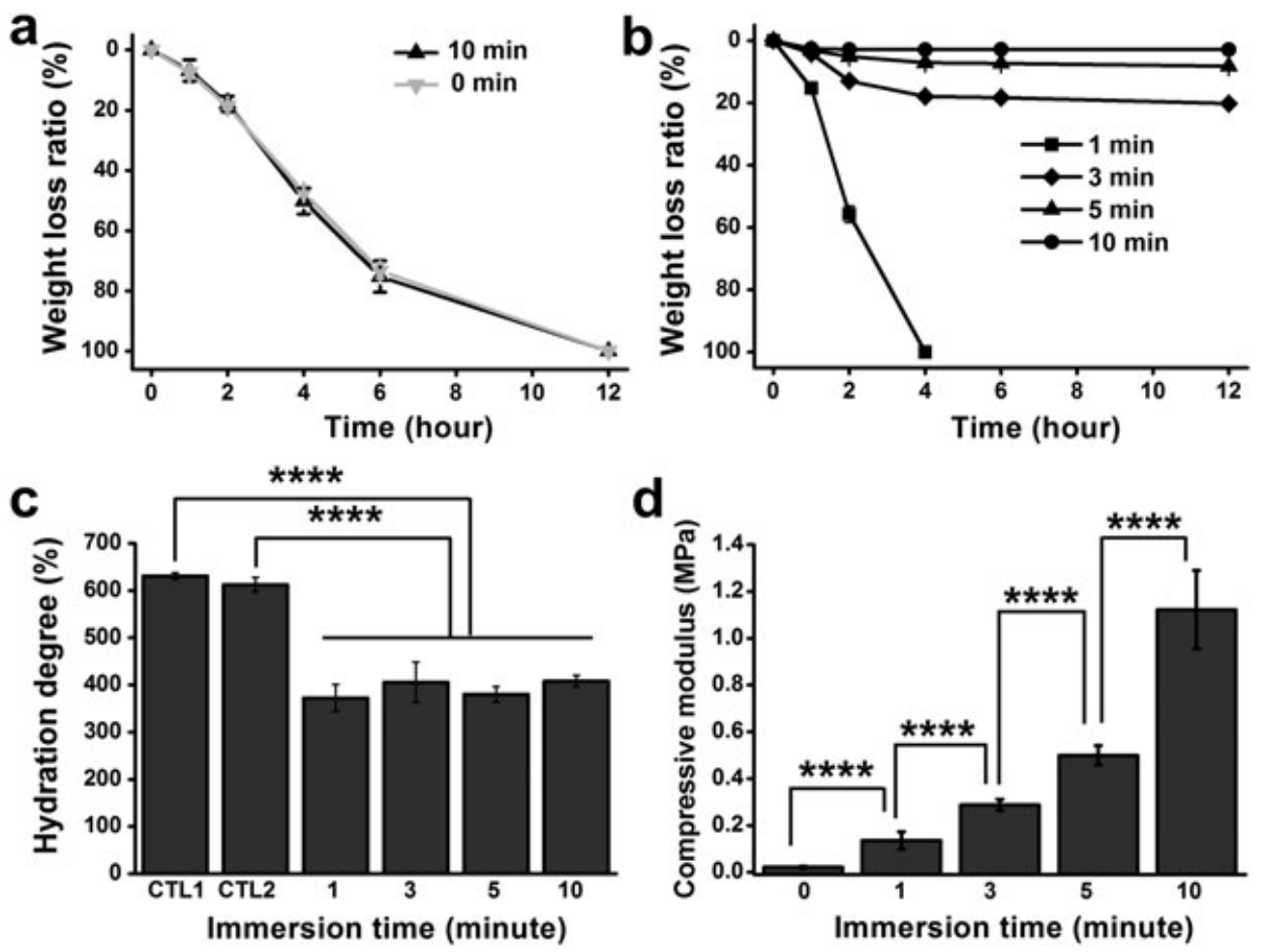

Figure 3. The enzymatic degradation profiles of (a) the core region, (b) the shell layer, (c) the hydration degree of the shell layer and (d) the compressive modulus of the coreshell silk fibroin (SF) hydrogels. (a, b) 0-10 min indicate the immersion time of hydrogels in methanol; the protease solution was $0.005 \mathrm{U} / \mathrm{ml}$ for (a) the core region and $0.2 \mathrm{U} / \mathrm{ml}$ for (b) the shell layer, $5 \mathrm{ml}$ solution was used for each specimen. (c, d) CTL1 and CTL2 correspond to the SF hydrogels without methanol treatment and the core region of the SF hydrogels after methanol treatment for $10 \mathrm{~min}$, respectively. ${ }^{* * * * *}$ Indicated statistically significant $(p<0.0001)$

Table 1. Weight remaining scores of the shell layer in the core-shell hydrogels

\begin{tabular}{llllll}
\hline $\begin{array}{l}\text { Methanol soaking } \\
\text { time, } \min \end{array}$ & \multicolumn{5}{c}{ Degradation time, $\mathrm{h}$} \\
\cline { 2 - 6 } & 1 & 2 & 4 & 6 & 12 \\
\hline $0^{*}$ & ++++ & +++ & ++ & + & - \\
$1^{\dagger}$ & +++ & ++ & - & & \\
$3^{+}$ & +++ & +++ & +++ & +++ & +++ \\
$5^{\dagger}$ & +++ & ++++ & ++++ & ++++ & ++++ \\
$10^{+}$ & ++++ & ++++ & ++++ & ++++ & ++++ \\
\hline
\end{tabular}

Almost no loss $(++++)$, slight loss $(+++)$, partial loss $(++)$, major loss $(+)$, complete loss (-).

*The samples were degraded in $0.005 \mathrm{U} / \mathrm{ml}$ protease XIV solution.

tThe samples were degraded in $0.2 \mathrm{U} / \mathrm{ml}$ protease XIV solution.

the hydrogel's mechanical performance was attributed to the increased thickness of the shell layer in the core-shell hydrogels.

\subsection{Controlled drug release}

In this study, albumin-FITC was used as a model drug and incorporated in the core-shell hydrogels. Its release profile was presented in Figure 4. The random coil SF hydrogels were able to incorporate $30.72 \pm 1.09 \mu \mathrm{g}$ of albumin per disc, and this amount was used for calculation of the incorporation efficiency of the core-shell hydrogels groups. After immersion in methanol for 1, 3, 5 and 10 $\min , 82.07 \pm 6.09 \%, 75.16 \pm 5.08 \%, 58.46 \pm 3.71 \%$ and $45.12 \pm 2.83 \%$ albumin remained in the core-shell SF hydrogel discs, respectively. As shown in Figure 4a and $\mathrm{b}$, the random coil SF hydrogels presented a lower intensity of fluorescence signal as compared with the core-shell hydrogels, after $24 \mathrm{~h}$. The cumulative drug release results showed that more than $60 \%$ of the incorporated albumin was released in the random coil SF hydrogels within $24 \mathrm{~h}$ (Figure 4c). After $72 \mathrm{~h}$, this group released nearly the total amount of the incorporated albumin. However, the core-shell SF hydrogels presented much slower albumin release profiles as compared with the random coil SF hydrogels (Figure 4c), except the group treated in methanol for $1 \mathrm{~min}$. The core-shell hydrogels treated with methanol for 1 min presented a slightly slower release profile compared with the random coil group in the first $6 \mathrm{~h}$, but no significant differences were found in the rest of the tested time points. In the first $24 \mathrm{~h}$, about $30 \%, 18 \%$ and $12 \%$ of the incorporated albumin was released from the core-shell SF hydrogels treated in methanol for 3, 5 and $10 \mathrm{~min}$, respectively. The release ratios of albumin in these three groups of hydrogels were comprised between $22 \%$ and $47 \%$ at the $72 \mathrm{~h}$ time point. One week later, it was observed that 30\%, 60\% and $70 \%$ of the incorporated albumin still remained in the core-shell SF hydrogels treated with methanol for 3, 5 and $10 \mathrm{~min}$, respectively.

\section{Discussion}

So far, varied approaches have been implemented to develop SF hydrogels of either $\beta$-sheet or random coil 

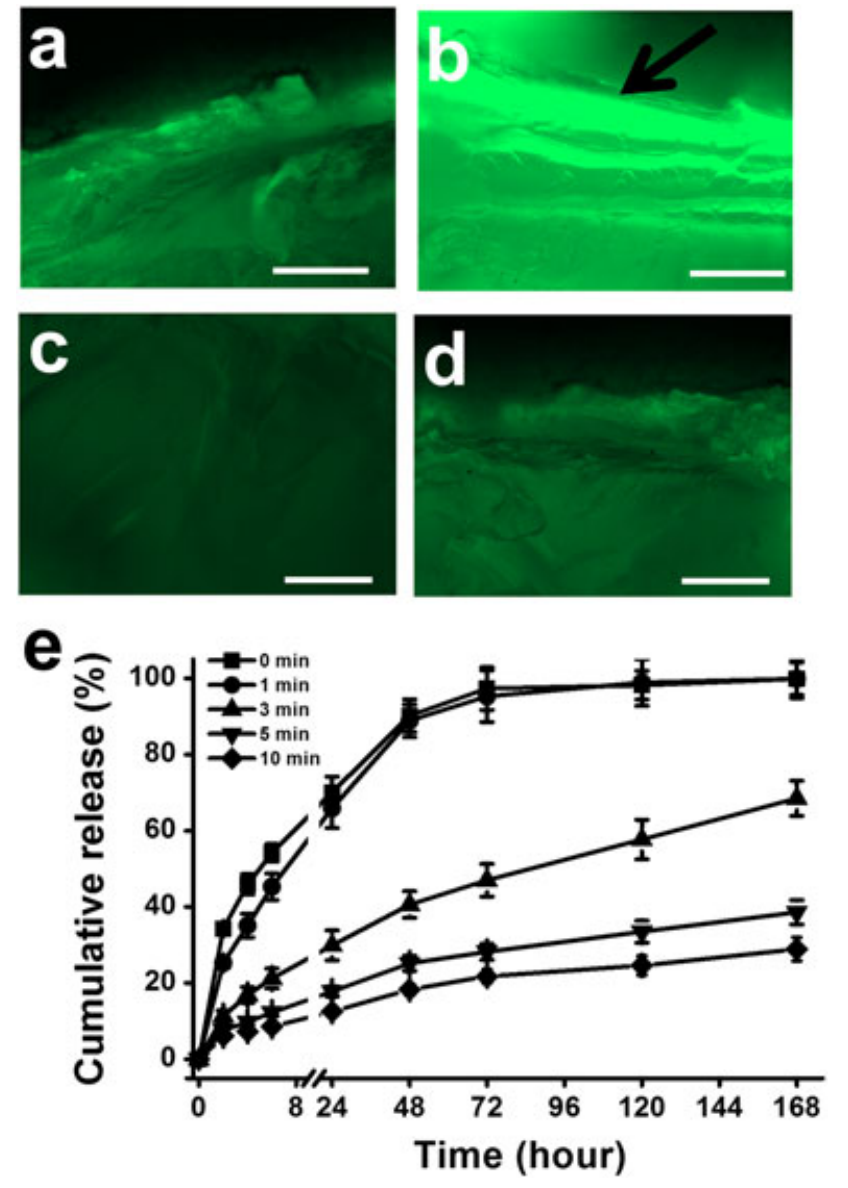

Figure 4. The albumin-fluorescein isothiocyanate (FITC) release profiles of the coreshell silk fibroin (SF) hydrogels. (a and b) Fluorescence images of the non-treated and the core-shell SF hydrogels (immersion for $3 \mathrm{~min}$ ) after releasing albumin for $24 \mathrm{~h}$, respectively. (c and d) Controls (without FITC incorporation hydrogels) for (a) and (b), respectively. Arrow indicated the outer layer of the layered SF hydrogels (scale bar: $300 \mu \mathrm{m}$ ). (e) Albumin-FITC release profile from the core-shell SF hydrogels after methanol treatment for 0 up to $10 \mathrm{~min}$

conformation (Leisk et al., 2010; Motta et al., 2004; Partlow et al., 2014; Wang et al., 2008; Yan et al., 2016). SF hydrogels proposed in those studies presented desirable in vitro and in vivo biocompatibility (Motta et al., 2004; Partlow et al., 2014; Yan et al., 2016), had been used for cell encapsulation (Partlow et al., 2014; Wang et al., 2008; Yan et al., 2016) or applied as bioadhesive (Leisk et al., 2010). All the above-mentioned SF hydrogels had macroscopically homogeneous properties and were lacking in spatially controlled physicochemical properties.

Organic solvents were able to induce fast $\beta$-sheet formation in SF from aqueous SF solution (Kundu and Kundu, 2013; Nazarov et al., 2004; Tamada, 2005). But due to the lack of a suitable platform, SF hydrogels of spatially controlled conformation had not been reported until now. In this study, we employed our previously developed random coil SF hydrogel as a platform (Yan et al., 2016), and combined methanol treatment to address this challenge. When the random coil SF hydrogels were immersed in methanol, the surface of the hydrogels would be dehydrated and immediately become a $\beta$-sheet. Thus, an opaque and stiff shell layer formed. As the dehydration happened gradually from the outer surface to the inner domain of the hydrogels, the thickness of this layer increased over time. However, the final thickness of the shell layer depends on the diffusion speed of methanol in the hydrogels and the immersion time in methanol. Because the hydrogels were only immersed in methanol for a few minutes, the core region was not affected by methanol yet and still maintained its original status. By using this easy but effective approach, SF hydrogels of core-shell structure can be obtained within minutes.

Different strategies have been reported for the development of core-shell hydrogels using different polymers (Abreu et al., 2009; Gan and Lyon, 2001; Iizawa et al., 2005; Koutsopoulos and Zhang, 2012; Ladet et al., 2008; Ma et al., 2013). In the study of Ladet et al., the shell layer was created through the neutralization of the charges in the initial alcohol gel, inducing the formation of a shell layer comprised of a physical gel and a core domain that still contained the alcohol gel (Ladet et al., 2008). Thermo-responsive polymers had been explored for core-shell hydrogels preparation. Gan et al. prepared poly-N-isopropylacrylamide core-shell nanoparticles via seed-feed precipitation polymerization (Gan and Lyon, 2001). Hydrogels composed of poly(N-alkylacrylamide) shell layer and poly(acrylic acid) core domain were generated through the selective amidation of poly(acrylic acid) gel as reported by Iizawa et al. (2005). Natural polymer or synthetic peptides were also used for manufacture of the core-shell structure of hydrogels. Alginate and Matrigel ${ }^{\circledR}$ core-shell microcapsules were developed by Ma et al. via a two-fluid co-axial electro-jetting method (Ma et al., 2013). Koutsopoulos and Zhang engineered selfassembling core-shell peptide hydrogels for long-term delivery of human antibodies (Koutsopoulos and Zhang, 2012). These core-shell hydrogel systems have been formed using different approaches: electrostatic force; two-stage polymerization of monomer(s); surfaceselective chemical reaction; ionic crosslinking; and self-assembly.

The current study provides a novel route to produce core-shell structural hydrogels, namely by controlling the spatial distribution of protein conformations. It is envisioned that the core-shell SF hydrogels, with spatially controlled characteristics, will present novel properties compared with the aforementioned SF systems, and will broaden the applications of SF in controlled drug delivery.

In order to fully elucidate the formation mechanism of the core-shell structure, we studied the conformation of SF in different domain of the hydrogels using ATR-FTIR in wet status. FTIR is a powerful tool to analyse the slight difference in SF conformation (Asakura et al., 1985; Yan et al., 2013). It has been mentioned that SF-based constructs were commonly composed of a mixture of different conformations (Guziewicz et al., 2011; Hu et al., 2011; Lu et al., 2010), even though their properties were determined by the dominant conformation. In order to increase their stability in water, crystallized structure was induced in SF, such as $\beta$-sheet or silk-I (Lu et al., 2010; Oliveira et al., 2012; Yan et al., 2012). Among the exceptions (non-crystallized structure), the electrically 
generated SF hydrogels and enzymatically crosslinked SF hydrogels were of dominant random coil structure (Leisk et al., 2010; Partlow et al., 2014; Yan et al., 2016).

During the immersion in methanol, the outer surface of the hydrogels would be immediately dehydrated. In dehydration status, the water molecules were repelled from the random coil SF molecules. Thus, the latter concentrated and formed $\beta$-sheet structure via self-assembly. This step was similar to the gel formation procedure by freezing the SF/solvent mixture (Tamada, 2005). This dehydration procedure would lead to the formation of an opaque and stiff shell layer. When methanol diffused into the hydrogels, it would show a gradient concentration decrease from the outer region to the inner region. Therefore, the dehydration level of the hydrogels gradually decreased from the external surface to the inner surface of the shell layer. The non-homogeneous dehydration level induced the gradient $\beta$-sheet content distribution in the shell layer. That was why the external surface of the shell layer displayed superior $\beta$-sheet content to the inner surface of the shell layer. In the case where the methanol concentration was lower than a certain level, the random coil hydrogel would be partially dehydrated and form a $\beta$-sheet gel paste, as mentioned in a previous study by Nazarov et al. (2004). The gel paste located between the shell layer of dominant $\beta$-sheet content and the non-affected core region acted as an interface region. This region was composed of both dominant $\beta$-sheet and random coil conformation. As the hydrogels were only immersed in methanol for 0-10 $\mathrm{min}$, the methanol did not diffuse to the core region yet during this short time period. Additionally, a shell layer formed immediately when the hydrogel was in contact with methanol, which subsequently hindered the diffusion of methanol into the inner domain. Moreover, the methanol was diluted in the diffusion procedure and removed as soon as the treatment was finalized by PBS. Therefore, the core region still maintained dominant random coil conformation.

Because there were obvious differences in the conformations of the core layer and the shell layer, these two layers presented distinct biodegradability and hydration properties. Because the core layer hydrogel was still of dominant random coil conformation, this layer degraded as fast as the non-treated hydrogels in the chosen protease solution concentration. When immersed in methanol for $1 \mathrm{~min}$, the shell layer treated by methanol for $1 \mathrm{~min}$ degraded much faster than other shell layer groups, due to its lower $\beta$-sheet content determined by the short immersion time. The longer immersion time in methanol favoured the stability of the shell layer, owing to the higher $\beta$-sheet transition ratio. Protease XIV was selected for the degradation test in this study. This enzyme is mainly used for the degradation of SF constructs of $\beta$-sheet structure (Yan et al., 2016). This explained why the core layer and non-treated hydrogels degraded so fast at such a low protease concentration. We used this protease for the degradation study as we aimed to show the distinct differences in biostability between the core layer and the shell layer. In the future, it would be helpful to use another protease to determine the random coil ratio in different layers in the core-shell hydrogels, such as $\alpha$-chymotrypsin which is specific for digestion of random coil domain in SF (Marelli et al., 2012). After $\beta$-sheet formation, the aggregated SF molecules in the shell layer became hydrophobic and the mesh size in the network decreased. Hence, the shell layers demonstrated much lower hydration degrees compared with the hydrophilic random coil core region and the non-treated hydrogels.

Another feature of the core-shell hydrogels is their widely tunable mechanical properties. The highly tailorable compressive modulus of the core-shell hydrogels came from the easily regulated $\beta$-sheet content in the shell layer. Previously, Kim et al. showed that the compressive modulus of SF hydrogels, which was induced by storage of aqueous SF solution (16\%) at different temperatures (room temperature, $37^{\circ} \mathrm{C}$ and $60{ }^{\circ} \mathrm{C}$ ), ranged from about 3 to $6 \mathrm{MPa}$ (Kim et al., 2004). In another study, Wang et al. prepared SF hydrogels by using sonication technique (Wang et al., 2008). The SF hydrogels, derived from 4 to $12 \%$ aqueous SF solution, displayed compressive modulus between 0.369 and $1.712 \mathrm{MPa}$. Compared with previous studies, the modulus of the core-shell hydrogels was lower than that reported by Kim et al. That is because the whole piece of thermalinduced hydrogel was of dominant $\beta$-sheet conformation, and the core-shell hydrogels only possessed dominant $\beta$-sheet content in the thin shell layer. However, time spent for the preparation of the core-shell hydrogels was much shorter than the thermally induced hydrogels. Because Wang et al. only used SF solution of less than $16 \%$ for the preparation of SF hydrogels, the modulus range of the sonication-induced hydrogels was comparable to that obtained in the current study.

It would be interesting to apply the mechanically tunable core-shell hydrogels for tissue substitute. The average compressive modulus of the human cartilage is about $1 \mathrm{MPa}$ (McMahon et al., 2008). After immersion in methanol for 5 and $10 \mathrm{~min}$, the core-shell SF hydrogels presented comparable moduli to human cartilage. Hence, these core-shell SF hydrogels display great potential as tissue substitutes for cartilage. When considering application of biodegradable materials for tissue replacement, the in vivo degradation behaviour is critical. In our previous study, the random coil SF hydrogels implanted subcutaneously in mice did not show obvious degradation after 4 weeks (Yan et al., 2016). In the current study, the shell layer showed superior stability to the random coil core layer. Based on these evidences, we deduced that the core-shell hydrogels showed desirable stability when applied as cartilage substitute in vivo.

Moreover, the core-shell hydrogels showed their great potential as drug-delivery systems. In random coil SF hydrogels, the SF molecules were highly hydrated and swelled, presenting large mesh size. Therefore, the incorporated albumin released very fast. In the shell layer of the core-shell SF hydrogels, the SF network became hydrophobic and shrank, forming a more compact 
structure and smaller mesh size compared with the random coil SF hydrogel. Consequently, the core-shell SF hydrogels displayed a slow and more controllable release profile of albumin as compared with the random coil SF hydrogels. The thicker shell layer allowed slower release of albumin from the core-shell SF hydrogels. As the shell layer after methanol treatment for 1 min was very thin and less stable compared with the shell layers from other core-shell hydrogel groups (Figures $1 \mathrm{~b}$ and $3 \mathrm{~b}$ ), this group presented a faster drug release profile than other groups. The stable release ratio of albumin in the core-shell hydrogels after 3-10 min treatment in methanol was related with the molecular weight of the albumin and the properties of the shell layer. After methanol treatment for 3-10 min, the shell layer became stable and thick. The molecular weight of albumin used herein is about $66 \mathrm{kDa}$. It would take a long time for these macromolecules to pass through the compact structure and small mesh size of the shell layer. Thus, the release profile was quite stable for these groups (methanol treated for 3-10 min).

The obtained results were consistent with previous studies (Choi et al., 2012; Koutsopoulos and Zhang, 2012). Koutsopoulos et al. found that double-layered hydrogels released the human antibodies slower than the single layer hydrogel, owing to the differences of the density and chemical properties of the two layer hydrogels (Koutsopoulos and Zhang, 2012). Choi et al. incorporated paclitaxel in multi-layered phospholipid polymer hydrogels, and found that the paclitaxel release depended on the location and concentration of the drugcontaining polymer layer (Choi et al., 2012). Different from the multi-component system or the time-consuming preparation procedures from aforementioned studies, this study only used one component and a very facile procedure to prepare the core-shell SF hydrogels. Therefore, the present study opens up the possibility of the core-shell SF hydrogels for application in the controlled release of growth factors or other bioactive macromolecules.

Incorporation of bioactive molecules into the scaffolds or hydrogels is interesting and promising in TERM (Srivastava et al., 2014). SF has been processed into carriers for delivery of drugs (Wenk et al., 2011). The herein proposed core-shell SF hydrogels are envisioned as a versatile and innovative system for controlled drug-delivery applications, such as for oral delivery of different therapeutic molecules. Nevertheless, this system will be evaluated further in the next step regarding its cytocompatiblity and the bioactivity of encapsulated drugs, as methanol is involved in the formation of the shell layer in the current study. Cytocompatibility will be studied by culturing cells on the core-shell hydrogels in the following study. The bioactivity of the encapsulated drugs will be studied by using growth factor or enzymes as model drugs in the next step (Wenk et al., 2008).

\section{Conclusion}

In this study, core-shell SF hydrogels with spatially controlled physicochemical properties were developed by spatial manipulation of the SF conformation. These hydrogels, comprised of a stiff-shell layer with main $\beta$-sheet conformation and soft inner region of dominant random coil conformation, were able to provide a sustained system for drug delivery. They further showed potential to be used as a cartilage substitute in terms of mechanical property. Overall, the core-shell SF hydrogels with spatially tailored structure open a new window for the application of SF-based biomaterials in TERM and as a drug-delivery system. This study also brings new insights in the development of protein-based biomaterials with sophisticated and hierarchical structures by tuning the protein conformation.

\section{Conflict of interest}

The authors have declared that there is no conflict of interest.

\section{Acknowledgements}

This study was funded by the Portuguese Foundation for Science and Technology (FCT) projects Tissue2Tissue (PTDC/CTM/ 105703/2008) and OsteoCart (PTDC/CTM-BPC/115977/ 2009), as well as the European Union's FP7 Programme under grant agreement no. REGPOT-CT2012-316331-POLARIS. Le-Ping Yan was awarded a FCT PhD scholarship (SFRH/BD/ 64717/2009). The FCT distinctions attributed to J.M. Oliveira and A.L. Oliveira under the Investigador FCT program (IF/ 00423/2012) and (IF/00411/2013) are also greatly acknowledged, respectively.

\section{References}

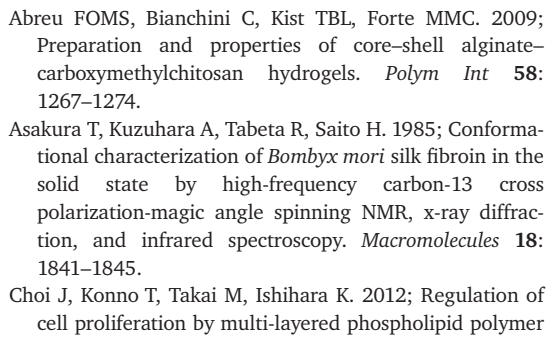

Choi J, Konno T, Takai M, Ishihara K. 2012; Regulation of cell proliferation by multi-layered phospholipid polymer

hydrogel coatings through controlled release of paclitaxel. Biomaterials 33: 954-961.

Fratzl P, Misof K, Zizak I et al. 1998; Fibrillar structure and mechanical properties of collagen. J Struct Biol 122: 119-122.

Gan D, Lyon LA. 2001; Tunable swelling kinetics in core shell hydrogel nanoparticles. J Am Chem Soc 123: 7511-7517.

Guziewicz N, Best A, Perez-Ramirez B, Kaplan DL. 2011; Lyophilized silk fibroin hydrogels for the sustained local delivery of therapeutic monoclonal antibodies. Biomaterials 32: $2642-2650$.

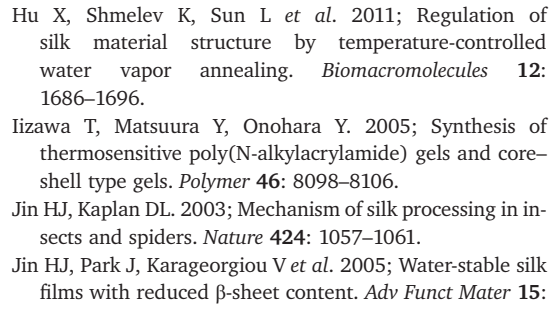

Hu X, Shmelev K, Sun L et al. 2011; Regulation of silk material structure by temperature-controlled water vapor annealing. Biomacromolecules 12 : 1686-1696.

Iizawa T, Matsuura Y, Onohara Y. 2005; Synthesis of thermosensitive poly(N-alkylacrylamide) gels and coreshell type gels. Polymer 46: 8098-8106.

Jin HJ, Kaplan DL. 2003; Mechanism of silk processing in insects and spiders. Nature 424: 1057-1061.

Jin HJ, Park J, Karageorgiou V et al. 2005; Water-stable silk films with reduced $\beta$-sheet content. Adv Funct Mater 15: 1241-1247. 


\section{L-P. Yan et al.}

Kim UJ, Park J, Li C et al. 2004; Structure and properties of silk hydrogels. Biomacromolecules 5: 786-792.

Kloxin AM, Kasko AM, Salinas CN, Anseth KS. 2009; Photodegradable hydrogels for dynamic tuning of physical and chemical properties. Science 324: 59-63.

Koutsopoulos S, Zhang S. 2012; Two-layered injectable selfassembling peptide scaffold hydrogels for long-term sustained release of human antibodies. J Control Release 160: 451-458.

Kundu B, Kundu SC. 2013; Bio-inspired fabrication of fibroin cryogels from the muga silkworm Antheraea assamensis for liver tissue engineering. Biomed Mater 8: 055003.

Kundu B, Kurland NE, Bano S et al. 2014; Silk proteins for biomedical applications: bioengineering perspectives. Prog Polym Sci 39: 251-267.

Ladet S, David L, Domard A. 2008; Multi-membrane hydrogels. Nature 452: 76-79.

Leisk GG, Lo TJ, Yucel T, Lu Q, Kaplan DL. 2010 Electrogelation for protein adhesives. Adv Mater 22: 711-715.

Lin Y, Xia X, Shang Ket al. 2013; Tuning chemical and physical cross-links in silk electrogels for morphological analysis and mechanical reinforcement. Biomacromolecules 14: 2629-2635.

Lu Q, Hu X, Wang X et al. 2010; Water-insoluble silk films with silk I structure. Acta Biomater 6: 1380-1387.

Ma L, Liu M, Liu H, Chen J, Cui D. 2010; In vitro cytotoxicity and drug release properties of $\mathrm{pH}$ - and temperaturesensitive core-shell hydrogel microspheres. Int J Pharm 385: 86-91.

Ma M, Chiu A, Sahay G et al. 2013; Core-shell hydroge microcapsules for improved islets encapsulation. Adv Healthc Mater 2: 667-672.

Makaya K, Terada S, Ohgo K, Asakura T. 2009; Comparative study of silk fibroin porous scaffolds derived 'from salt/water and sucrose/hexafluoroisopropanol in cartilage formation. J Biosci Bioeng 108: 68-75.
Marelli B, Ghezzi CE, Alessandrino A et al. 2012; Silk fibroin derived polypeptide-induced biomineralization of collagen. Biomaterials 33: 102-108.

McMahon LA, O'Brien FJ, Prendergast PJ. 2008; Biomechanics and mechanobiology in osteochondral tissues. Regen Med 3: 743-759.

Motta A, Migliaresi C, Faccioni F et al. 2004; Fibroin hydrogels for biomedical applications: preparation, characterization and in vitro cell culture studies. $J$ Biomater Sci Polym Ed 15: 851-864.

Nazarov R, Jin HJ, Kaplan DL. 2004; Porous 3-D scaffolds from regenerated silk fibroin. Biomacromolecules 5: 718-726.

Nowak AP, Breedveld V, Pakstis L et al. 2002; Rapidly recovering hydrogel scaffolds from self-assembling diblock copolypeptide amphiphiles. Nature 417: 424-428.

Numata K, Yamazaki S, Naga N. 2012; Biocompatible and biodegradable dual-drug release system based on silk hydrogel containing silk nanoparticles. Biomacromolecules 13: 1383-1389.

Oliveira AL, Sun L, Kim HJ et al. 2012; Aligned silk-based 3 $\mathrm{D}$ architectures for contact guidance in tissue engineering. Acta Biomater 8: 1530-1542.

Partlow BP, Hanna CW, Rnjak-Kovacina J et al. 2014; Highly tunable elastomeric silk biomaterials. Adv Funct Mater 24: 4615-4624.

Pearle AD, Warren RF, Rodeo SA. 2005; Basic science of articular cartilage and osteoarthritis. Clin Sports Med 24: 1-12.

Reis RL, Yan LP, Oliveira AL et al. 2014; Hydrogels derived from silk fibroin: methods and uses thereof. A4TEC Association, assignee. Portuguese patent PT107426.

Seib FP, Pritchard EM, Kaplan DL. 2013; Self-assembling doxorubicin silk hydrogels for the focal treatment of primary breast cancer. Adv Funct Mater 23: 58-65.

Seliktar D. 2012; Designing cell-compatible hydrogels for biomedical applications. Science 336: 1124-1128.
Silva R, Fabry B, Boccaccini AR. 2014; Fibrous protein-based hydrogels for cell encapsulation. Biomaterials 35 6727-6738.

Srivastava A, O'Connor IB, Pandit A, Gerard Wall J. 2014, Polymer-antibody fragment conjugates for biomedical applications. Prog Polym Sci 39: 308-329.

Tamada Y. 2005; New process to form a silk fibroin porous 3-D structure. Biomacromolecules 6: 3100-3106.

Vepari C, Kaplan DL. 2007; Silk as a biomaterial. Prog Polym Sci 32: 991-1007.

Wang X, Kluge JA, Leisk GG, Kaplan DL. 2008; Sonicationinduced gelation of silk fibroin for cell encapsulation. Biomaterials 29: 1054-1064.

Wenk E, Merkle HP, Meinel L. 2011; Silk fibroin as a vehicle for drug delivery applications. $J$ Control Release 150: $128-141$.

Wenk E, Wandrey AJ, Merkle HP, Meinel L. 2008; Silk fibroin spheres as a platform for controlled drug delivery. J Control Release 132: 26-34.

Yan LP, Silva-Correia J, Ribeiro VP et al. 2016; Tumo growth suppression induced by biomimetic silk fibroin hydrogels. Sci. Rep 6: 31037.

Yan LP, Oliveira JM, Oliveira AL et al. 2012; Macro/microporous silk fibroin scaffolds with potential for articular cartilage and meniscus tissue engineering applications. Acta Biomater 8: 289-301.

Yan LP, Oliveira JM, Oliveira AL, Reis RL. 2015; In vitro evaluation of the biological performance of macro/micro-porous silk fibroin and silk-nano calcium phosphate scaffolds. J Biomed Mater Res B Appl Biomater 103: 888-898.

Yan LP, Salgado AJ, Oliveira JM, Oliveira AL, Reis RL. 2013; De novo bone formation on macro/microporous silk and silk/nano-sized calcium phosphate scaffolds. $J$ Bioact Compat Pol 28: 439-452.

Yucel T, Cebe P, Kaplan DL. 2009; Vortex-induced injectable silk fibroin hydrogels. Biophys $J$ 97: 2044-2050. 